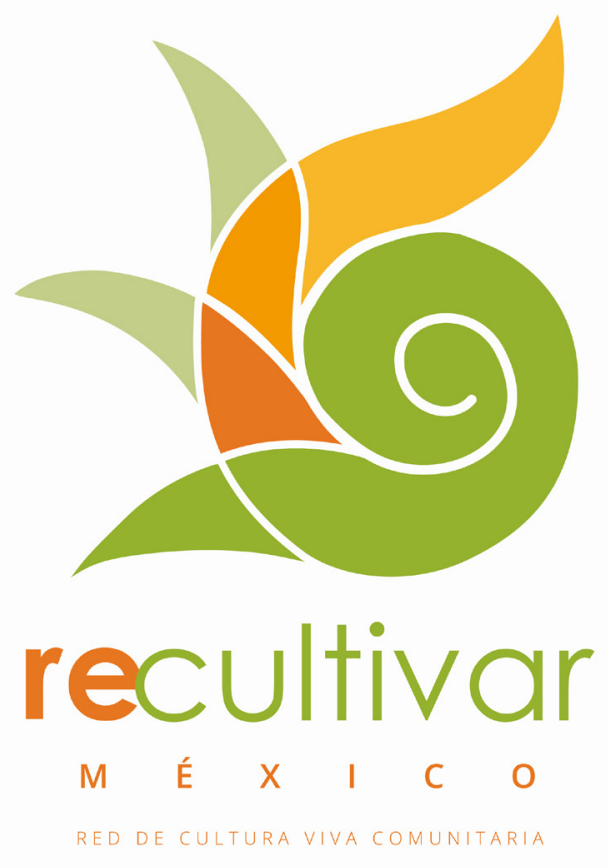

\title{
Gestores culturales: dónde están y qué hacen en las altas montañas de Veracruz Recultivar México, Red de Cultura Viva Comunitaria
}

M. L. Becerra ${ }^{1}$ - F. Colin²

RESUMEN: Recultivar México, Red de Cultura Viva Comunitaria, es una iniciativa que vincula a algunos gestores de la región Córdoba-Orizaba, México, con el Observatorio de Políticas Culturales de la Universidad Veracruzana (OPC). Realizada con la propuesta metodológica de la cibercultur@, busca construir un sistema de información cultural que pueda servir para un análisis, desde abajo, de la Política Cultural. Los datos presentados corresponden a los proporcionados por los gestores que se han suscrito a Recultivar de junio a diciembre de 2017. El siguiente paso de Recultivar es fortalecer el sistema de comunicación para ampliar el sistema de información y conocimiento.

Palabras Clave: gestión cultural, cibercultur@, política cultural, sistema de información, sistema de comunicación.
ABSTRACT: Recultivar México, Red de Cultura Viva Comunitaria, is an initiative that links some cultural managers of the Córdoba-Orizaba region, with the Observatory of Cultural Policies of the Universidad Veracruzana (OPC). Carried out with the methodological proposal of the cibercultur@, aims to build a cultural information system that can support an analysis, from below, of Cultural Policy. The data presented correspond to those provided by the cultural managers who have subscribed to Recultivar from June to December 2017. The next step of Recultivar is to strengthen the communication system to expand the information and knowledge system.

Keywords: cultural management, cibercultur@, cultural policy, communication system, knowledge system.

1 lbecerra@uv.mx Facultad de Antropología de la Universidad Veracruzana. Observatorio de Políticas Culturales.

2 federicocolin@gmail.com Facultad de Antropología de la Universidad Veracruzana. Observatorio de Políticas Culturales. 
Introducción

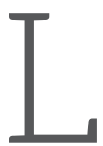
a intención de comunicar y compartir experiencias de trabajo de los distintos observatorios de la Universidad Veracruzana en éste espacio, hace posible enriquecer y retroalimentar la misión de cada uno. Divulgar sus acciones, participantes, eventos y avatares, serán el resultado de un esfuerzo colectivo, esto es comprensible ya que es una producción colaborativa que busca, poco a poco, dotar de información con indicadores, que oriente a nuestra comunidad universitaria en un principio y sociedad en general, para la toma de decisiones de los responsables de las políticas públicas

En el estado de Veracruz, y en todo el país, México, existe una diversidad de redes de intercambio de información que se hospedan y difunden a través de la Internet, consideramos que es significativo fortalecer el quehacer de nuestras organizaciones culturales articulando actividades y generando un gran movimiento nacional, apoyados en las Tecnologías de Información y Comunicación (TIC), lo suficientemente congruente y sistemático para incidir desde la perspectiva de la sociedad civil organizada, en las políticas culturales de nuestro país, nuestros estados, nuestros municipios y nuestras comunidades.

En abril de 2017 después de ocho meses de pláticas y acuerdos, el Observatorio de Políticas Culturales (OPC) de la Facultad de Antropología UV, la Coordinación Universitaria de Observatorios (CUO) de la UV, Universidad Veracruzana Intercultural, Nodo Sur Incubadora Cultural y Laboratorio Escénico A.C. (Labeac), lanzamos la convocatoria para integrar Recultivar México, Red de Cultura Viva Comunitaria, con una respuesta de más de 50 organizaciones, artistas y colectivos registrados, quienes nos dimos cita para platicar e intercambiar experiencias, con el propósito de identificar necesidades e intereses que orienten la constitución de una primera agenda que fomente y fortalezca el trabajo colaborativo como ejercicio incluyente y distintivo, así como para generar un sistema de información que recupere la trayectoria y experiencias de gestores, artistas, promotores y emprendedores culturales.

El objetivo general de Recultivar México, Red de Cultura Viva Comunitaria, es la articulación formal de gestores, artistas, agrupaciones, organizaciones y espacios, favoreciendo las condiciones de ejercicio de los derechos culturales de las comunidades donde se desarrollan los miembros de la red.

La misión se orienta a sistematizar, articular y fortalecer los intercambios de conocimientos, actividades de agentes que trabajan a favor y con la cultura, para reconocer y favorecer el ejercicio de los derechos culturales. Así mismo, busca constituirse como un espacio de intercambio de comunicación a partir de la información proporcionada por diversos agentes que trabajan a favor y con la cultura, mediante un sistema de información en Internet, del cual emerjan las actividades en favor del ejercicio de los derechos.

Orientaciones generales: definiciones y procedimientos

Lo que se percibe como Política Pública se refiere al horizonte en el cual las autoridades públicas señalan propósitos y programas que buscan alcanzar ciertos objetivos, necesarios o deseados, que buscan modificar un estado de cosas considerado problemático (Roth, 2002). Se espera que las autoridades públicas, sean las responsables de estructurar sistemática y establemente las acciones que atienden intereses públicos. Sin embargo, en la constitución y proceso de estructuración de las Políticas Públicas (PP) están involucrados agentes y autoridades no gubernamentales con los que se puede dialogar para convenir, negociar o reconocer: instrumentos legales y administrativos, recursos humanos y económicos (Aguilar, 2010).

Particularmente, la Política Cultural sería el conjunto de iniciativas tomadas por diversos agentes como el Estado, instituciones civiles, entidades privadas o grupos comunitarios, con el objeto de promover la producción, distribución y disfrute de la cultura, así como la preservación y divulgación de sus patrimonios (Coelho, 2009, p. 241). El enfoque general es del 
análisis de la Política Cultural desde abajo, desde las prácticas e interacciones sociales que se desarrollan día a día, cara a cara, y que proveen de sentido a los escenarios sociales que se reconocen como el mundo de la vida. A través de prácticas de sentido, acciones que construyen esos escenarios sociales, se pueden localizar quiénes, dónde, cómo, por qué, para qué, cómo, con qué y cuándo se articulan las redes sociales que definen los elementos de la agenda pública, en este caso, relacionado con la cultura.

Esta perspectiva desde abajo la asumimos en la figura del gestor cultural. Ante la diversidad de acciones y espacios en los que ésta figura se desenvuelve, no es sencillo dar una definición. En buena medida, la figura del gestor es definida por las concepciones de cultura que acompañan sus acciones. Con la finalidad de delimitar de manera general qué entendemos por gestor cultural, asumimos que es la figura que busca dar resolución a problemas indivi- duales y/o colectivos, estimulando la creación y transformación social a partir de sus relaciones con otras personas como intelectuales, artistas, funcionarios, e incluso otros gestores culturales (Román, 2011).

El modelo seguido para el reconocimiento de las situaciones públicas que pueden y debieran ser consideradas para la agenda de la Política Cultural es de movilización (Roth, 2002) por parte de mediadores sociales que se constituyen como legítimos a través de su actividad social organizada: los gestores culturales. Su legitimidad en la implementación y evaluación de las Políticas Culturales en Iberoamérica está reconocida por diversos autores como Jiménez (2006), y Román (2011) y Mariscal (2006). Por ello hemos partido de la perspectiva de análisis propuesta por el último autor para ubicar la diversidad de los gestores con quienes hemos trabajado:

Imagen 1. Clasificación de visiones de la gestión cultural. Mariscal (2006, p. 61)

\begin{tabular}{|c|c|c|c|}
\hline Aspectos & Área social & Área administrativa & Área artística \\
\hline Visión de la cultura & $\begin{array}{l}\text { Conjunto de sistemas sim- } \\
\text { bólicos que representa la } \\
\text { relación del hombre con } \\
\text { sus semejantes, la natura- } \\
\text { leza y el cosmos reflejados } \\
\text { en conocimientos, creen- } \\
\text { cias, costumbres, usos y } \\
\text { hábitos sociales. }\end{array}$ & $\begin{array}{l}\text { Servicios que se dan a } \\
\text { partir de la oferta y la } \\
\text { demanda }\end{array}$ & $\begin{array}{l}\text { Manifestaciones } \\
\text { artísticas }\end{array}$ \\
\hline Visión de la práctica & $\begin{array}{l}\text { Serie de actividades en- } \\
\text { caminadas a desatar y } \\
\text { acompañar procesos de } \\
\text { desarrollo social }\end{array}$ & $\begin{array}{l}\text { Desarrollo de proyectos } \\
\text { eficientes y eficaces }\end{array}$ & $\begin{array}{l}\text { Acciones encaminadas a } \\
\text { la difusión y educación } \\
\text { artística }\end{array}$ \\
\hline Papel del promotor & $\begin{array}{l}\text { Agenmbiote de cambio } \\
\text { social }\end{array}$ & $\begin{array}{l}\text { Administrador } \\
\text { cultural }\end{array}$ & $\begin{array}{l}\text { Mediador entre el arte y } \\
\text { la sociedad }\end{array}$ \\
\hline $\begin{array}{l}\text { Unidad de producción } \\
\text { predilecta }\end{array}$ & $\begin{array}{l}\text { Proyectos de investiga- } \\
\text { ción, la capacitación cul- } \\
\text { tural y el desarrollo comu- } \\
\text { nitario }\end{array}$ & $\begin{array}{l}\text { Actividades artísticas, } \\
\text { desarrollo de la infraes- } \\
\text { tructura cultural, aná- } \\
\text { lisis de públicos y de la } \\
\text { economía de la cultura, } \\
\text { campañas de gestión de } \\
\text { recursos y proyectos de } \\
\text { difusión cultural }\end{array}$ & $\begin{array}{l}\text { Las actividades artísti- } \\
\text { cas, exposiciones, edu- } \\
\text { cación artística, y en } \\
\text { general actividades de } \\
\text { acercamiento al arte }\end{array}$ \\
\hline
\end{tabular}


A partir de lo anterior, se estableció la clasificación como:

- Gestores (realizan cualquiera de las tres actividades siguientes, principalmente desde la administración)

- Emprendedores culturales (orientan su actividad hacia la actividad empresarial)

- Promotores/animadores culturales (trabajan principalmente hacia el área social)

- Creadores (se asumen como artistas que pueden orientarse hacia la empresa y/o el área social).

Esto permite identificar áreas de interés relacionados con el ejercicio de los derechos culturales, pues en la medida que los gestores trabajan sobre la base de la legitimidad social de base comunitaria y asociación entre ellos, pueden observarse una serie de necesidades de tipo socio-cultural atendidas por ellos.

\section{Organizando y comunicando la información}

Las preguntas sobre la información que nos ayude a diseñar, evaluar, implementar adecuadamente las Políticas Culturales no son asunto menor, implica el reconocimiento de las maneras de relacionarse entre los diversos agentes involucrados en cuanto a intereses, preocupaciones, definiciones de problemas o situaciones susceptibles de mejora y que son de interés público.

La manera en cómo organizamos la información que requerimos, cómo somos capaces de interactuar a través de ella, y generar un crecimiento de esa información en conocimiento, a través de la tecnología que disponemos, es una manera de cultivar otras formas de relacionarnos, más horizontales y cercanas a las comunidades directamente involucradas en los asuntos públicos.

En particular para los gestores implica reconocer dónde y cómo obtienen, organizan y resguardan la información relacionada con la cultura que desean gestionar; cómo y con quiénes traducen esa información en conocimiento, esto es, en una guía para la acción lo suficientemen- te sólida y amplia que trascienda un caso particular; y cuáles son las interacciones (con quiénes, dónde, cómo, por qué, para qué, cuándo) que les permiten, han permitido y permitirán llevar a cabo la gestión cultural.

Los gestores podrían evaluar, además de sus propios sistemas de información, cuáles son aquellos que les permiten acceder de manera rápida para el diseño, evaluación y prospectiva de su ejercicio profesional, de tal manera que sea posible re-construir la memoria de quiénes somos, qué hemos hecho; comprender el presente y prefigurar escenarios de acción.

El siguiente paso es establecer los sistemas de comunicación, los cuales se basan en la reorganización de las acciones y relaciones. La posibilidad de crear la propia organización, ratificar las normas y principios de esas relaciones, regular el intercambio de información, productos, y fomentar los valores que garanticen la existencia del grupo hacia el futuro, orientan el desarrollo sostenible de las comunidades (Maass \& Recaman, 2014).

Un esquema de la metodología que el Observatorio de Políticas Culturales ha trazado para la articulación de Recultivar México se puede apreciar en la imagen 2.

\section{Imagen 2. Esquema de cibercultur@. Elaborado a partir de Maass y Amozurrutia (2013)}

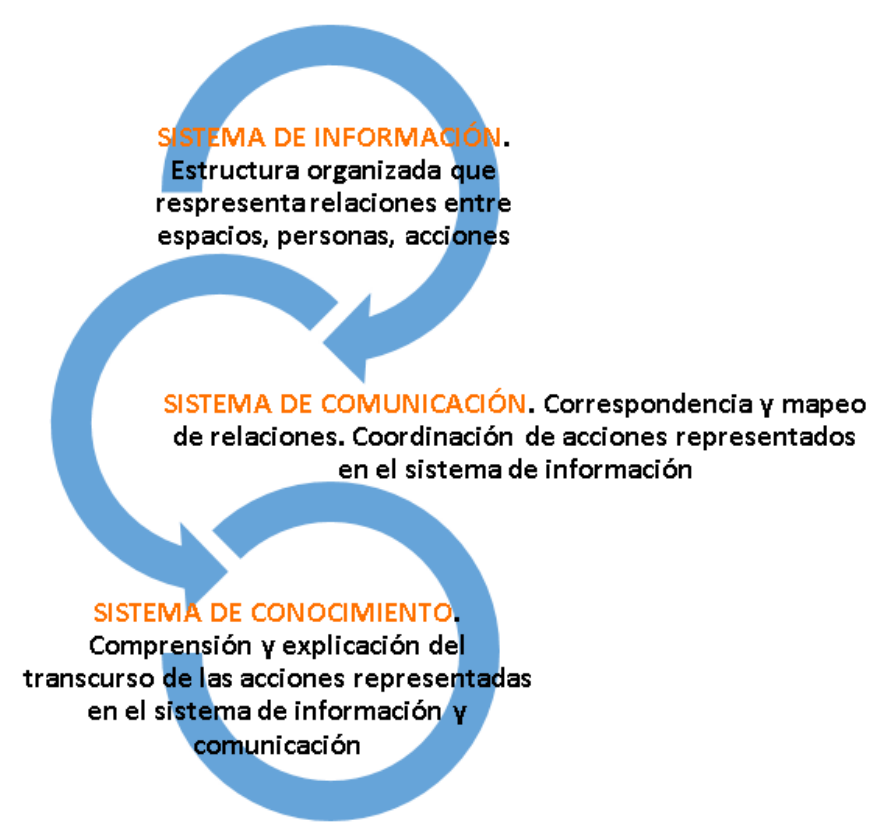


De esta forma, Recultivar México puede reconocerse en al menos dos tipos de conjuntos de acciones en los que el OPC es un mediador:

1. La articulación de los gestores en sus acciones, espacios, problemas e intereses. Es la fuente de información principal de Recultivar y tiene su propia lógica de sistema de comunicación

2. La organización de la información generada por los gestores por parte del OPC. Tiene un sistema de comunicación diferente al punto 1, y se articula con la Coordinación Universitaria de Observatorios (CUO) para su divulgación.

El primer informe Recultivar México, Red de Cultura Viva Comunitaria.

El informe, realizado en enero de 2018, está basado en la metodología de la cibercultur@, ya explicado anteriormente, y en el análisis general de la Política Pública Cultural, pensada de abajo hacia arriba. Es un primer diagnóstico que presenta la información que los gestores amablemente han proporcionado al OPC, y con lo que esperamos construir nuestro sistema de comunicación sobre el ejercicio de los derechos culturales. Algunos de los datos más relevantes se presentan en la tabla $1^{3}$.

La tabla anterior incorpora el formulario elaborado por el equipo de la CUO, y se puede consultar en https://www.uv.mx/apps/cuo/ opc/recultivar_mexico/. Es la página de registro de Recultivar y se ha pilotado con 10 de los miembros. Se acordó con los gestores que como datos generales eran suficientes para el registro, y se valoraría más adelante si es necesario ampliarlo o reducirlo.

Los datos que se presentan en el gráfico 1 corresponden al formulario inicial que se realizó en un formulario de google, en junio de 2017.

Dos aspectos nos parecen fundamentales, reconocer dónde desarrollan los gestores su labor, y la población objetivo de sus actividades. Esto permitirá, en primera instancia, localizarlos de acuerdo a su tipo de gestión, y comenzar a delimitar lo que ellos asumen como problemas públicos, a partir de las personas y actividades desarrolladas.

Se tiene un primer mapa de localización por tipos de gestión cultural. Se puede apreciar un mapa sensible con los datos de identificación de cada gestor en https://www.uv.mx/redrecultivarmexico/miembros-de-la-red/miembros-de-la-red-recultivar-mexico/.

\section{Gráfico 1. Miembros Recultivar México por Estado de la República Mexicana (2017)}

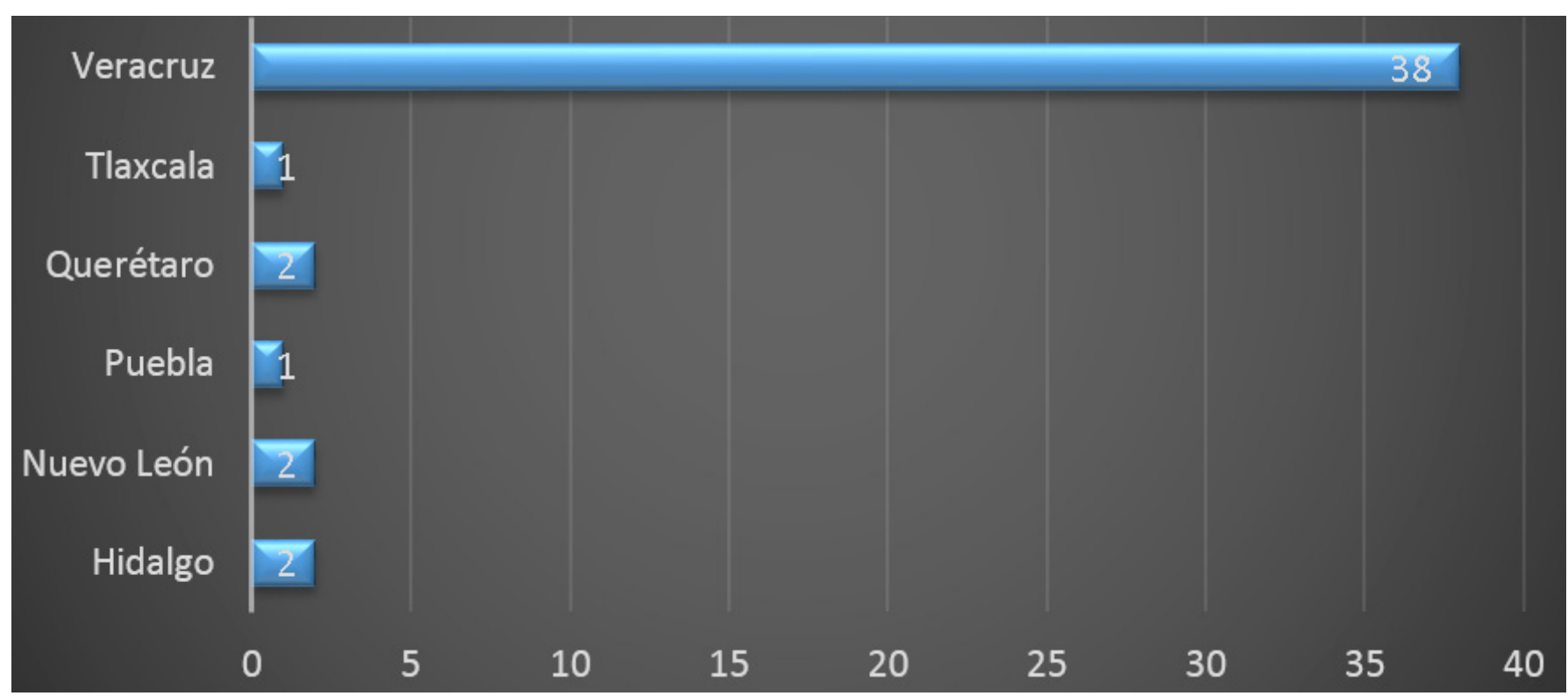

3 El informe completo puede consultarse en wWW.uv.mx/opc. 


\begin{tabular}{|c|c|c|c|c|}
\hline Datos generales & $\begin{array}{l}\text { Antecedentes } \\
\text { y principales } \\
\text { problemas }\end{array}$ & Recursos humanos & $\begin{array}{l}\text { Recursos } \\
\text { materiales }\end{array}$ & $\begin{array}{l}\text { Recursos } \\
\text { financieros }\end{array}$ \\
\hline $\begin{array}{l}\text { Tipo de } \\
\text { Organización }\end{array}$ & $\begin{array}{l}\text { Año de } \\
\text { Fundación }\end{array}$ & $\begin{array}{l}\text { Número de integrantes } \\
\text { permanentes }\end{array}$ & $\begin{array}{l}\text { Infraestructura } \\
\text { de la } \\
\text { Organización }\end{array}$ & $\begin{array}{l}\text { Principales fuentes } \\
\text { de financiamiento }\end{array}$ \\
\hline $\begin{array}{l}\text { Nombre de la } \\
\text { Organización }\end{array}$ & Financiamiento & $\begin{array}{l}\text { Principales funciones des- } \\
\text { empeñadas por los } \\
\text { integrantes permanentes }\end{array}$ & & $\begin{array}{l}\text { Beneficio de algún } \\
\text { programa en los úl- } \\
\text { timos } 3 \text { años }\end{array}$ \\
\hline $\begin{array}{l}\text { Nombre del Coor- } \\
\text { dinador General }\end{array}$ & $\begin{array}{l}\text { Falta de } \\
\text { colaboradores }\end{array}$ & $\begin{array}{l}\text { Número de integrantes } \\
\text { que desempeñan activida- } \\
\text { des laborales externas a la } \\
\text { organización }\end{array}$ & & $\begin{array}{l}\text { Nombre del } \\
\text { programa }\end{array}$ \\
\hline Estado & $\begin{array}{l}\text { Zona de trabajo } \\
\text { lejana de } \\
\text { centros urbanos }\end{array}$ & $\begin{array}{l}\text { Cuenta con colaboradores } \\
\text { eventuales }\end{array}$ & & $\begin{array}{l}\text { Tipo de beneficio: } \\
\text { Equipamiento }\end{array}$ \\
\hline Municipio & $\begin{array}{l}\text { Zona de trabajo } \\
\text { de alta } \\
\text { peligrosidad }\end{array}$ & $\begin{array}{l}\text { Tipo de colaboración: } \\
\text { Apoyo de especialista }\end{array}$ & & $\begin{array}{l}\text { Tipo de beneficio: } \\
\text { Colaboración de es- } \\
\text { pecialistas }\end{array}$ \\
\hline Localidad & & $\begin{array}{l}\text { Tipo de colaboración: } \\
\text { Elaboración de materiales } \\
\text { de trabajo }\end{array}$ & & $\begin{array}{l}\text { Tipo de beneficio: } \\
\text { Recurso material }\end{array}$ \\
\hline $\begin{array}{l}\text { Correo } \\
\text { Electrónico }\end{array}$ & & $\begin{array}{l}\text { Tipo de colaboración: } \\
\text { Infraestructura }\end{array}$ & & $\begin{array}{l}\text { Tipo de beneficio: } \\
\text { Recurso económico }\end{array}$ \\
\hline Página Web & & $\begin{array}{l}\text { Tipo de colaboración: } \\
\text { Gestión en comunidad }\end{array}$ & & \\
\hline $\begin{array}{l}\text { Página de } \\
\text { Facebook }\end{array}$ & & Cursos-talleres & & \\
\hline Página de Twitter & & Festivales & & \\
\hline $\begin{array}{l}\text { Alcance } \\
\text { Territorial }\end{array}$ & & $\begin{array}{l}\text { Foros/Seminarios/Colo- } \\
\text { quios }\end{array}$ & & \\
\hline $\begin{array}{l}\text { Objetivo } \\
\text { Principal }\end{array}$ & & $\begin{array}{l}\text { Presentaciones/Exposi- } \\
\text { ciones }\end{array}$ & & \\
\hline $\begin{array}{l}\text { Población } \\
\text { Objetivo }\end{array}$ & & $\begin{array}{l}\text { Logística de producción } \\
\text { artística }\end{array}$ & & \\
\hline \multirow[t]{2}{*}{$\begin{array}{l}\text { Población } \\
\text { Objetivo Otro }\end{array}$} & & Curaduría/restauración & & \\
\hline & & $\begin{array}{l}\text { Desarrollo/integración/ } \\
\text { aplicación de TIC para ac- } \\
\text { tividades y servicios cul- } \\
\text { turales }\end{array}$ & & \\
\hline
\end{tabular}


Tabla 2. Número de gestores por tipo de población objetivo

\begin{tabular}{|c|c|c|c|c|c|c|}
\hline Jóvenes & Infantes & Mujeres & $\begin{array}{c}\text { Público en } \\
\text { general }\end{array}$ & $\begin{array}{c}\text { Adultos } \\
\text { mayores }\end{array}$ & $\begin{array}{c}\text { Población } \\
\text { situación de } \\
\text { calle }\end{array}$ & $\begin{array}{c}\text { Gestores } \\
\text { culturales }\end{array}$ \\
\hline 27 & 20 & 16 & 15 & 14 & 6 & 4 \\
\hline
\end{tabular}

Mapa 2. Tipos de gestión de las Altas Montañas de Veracruz, Recultivar México, Red de Cultura Viva Comunitaria

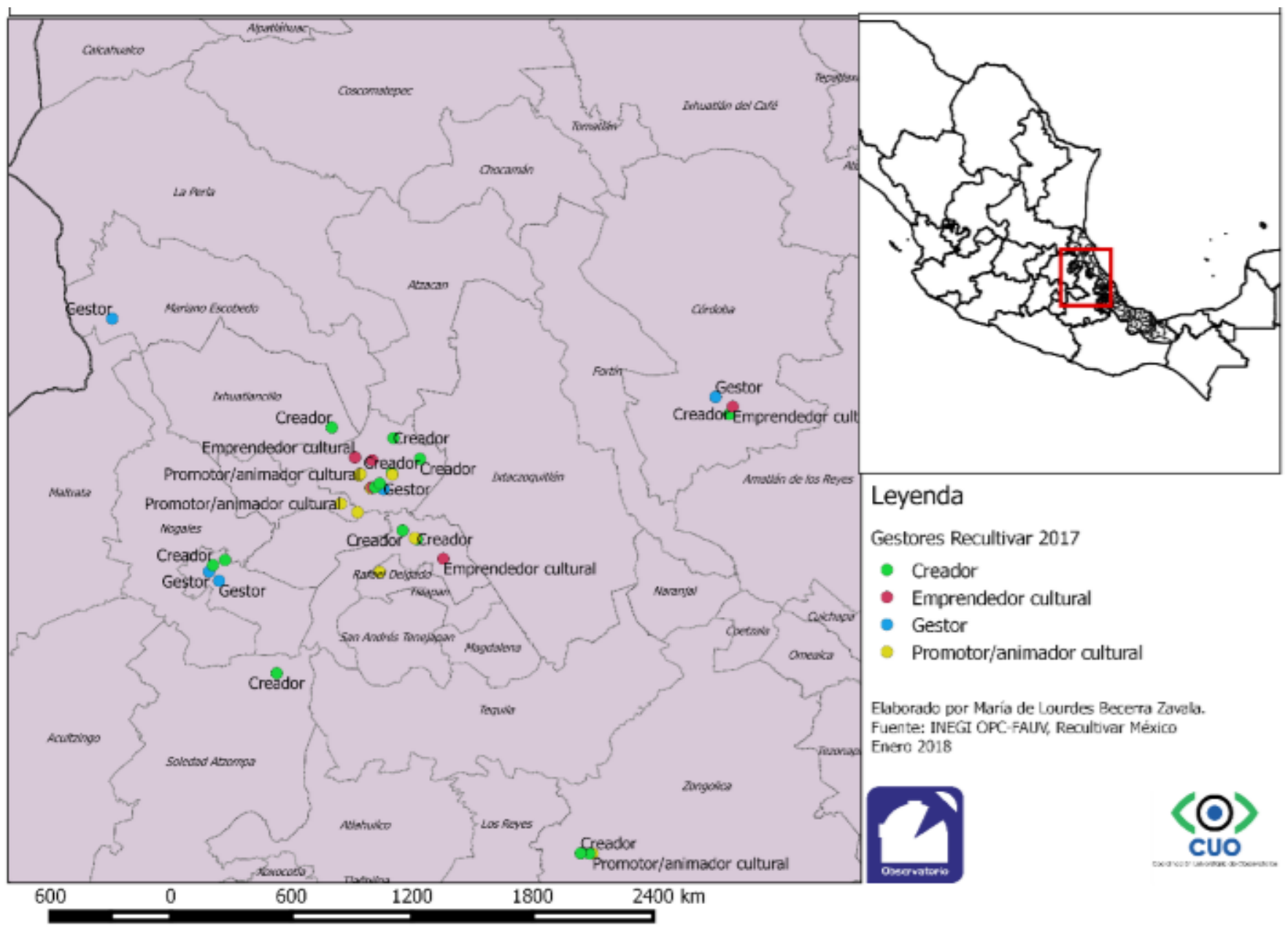

Fuente: elaborado por María de Lourdes Becerra Zavala con información del INEGI, OPC FAUV, Recultivar México. (Enero 2018)

Reflexiones finales

En el OPC consideramos que uno de sus propósitos debe consistir en acortar el distanciamiento entre los tomadores de decisiones sobre políticas culturales y los agentes involucrados en su desarrollo e investigación. Muchas veces desde la academia se pueden criticar (y esa es una labor importante, clave de las ciencias sociales) los diseños de políticas culturales, sin embargo "¿dónde está la información, los bancos de información densos, llenos de relaciones, llenos de elementos prácticas que permitirían hacer -y no improvisar- el seguimiento y la evaluación de su propia acción en políticas culturales?" (González, 2007, p. 50). Para ello es 
necesario el cultivo de la información que puede definirse como la capacidad de re-producir la memoria, comprender el presente y prefigurar posibles escenarios de manera diseñada, sistémica para mantener, distribuir información y generar el diseño, la evaluación y prospectiva de las actividades de gestión cultural es el cultivo de información (González, 2007).

Consideramos que es fundamental -en primera instancia- que los gestores culturales sean quienes produzcan y ponderen la información relevante para sus actividades cotidianas: qué hacen, con quiénes, qué es lo indispensable para su labor. En segundo lugar, que esa información puedan recuperarla, tenerla a su disposición de manera oportuna y eficiente, y se vuelva un elemento de comunicación que genere lazos sociales comunitarios con otros gestores y con las poblaciones objetivo con quienes desarrollan sus actividades. Finalmente, la información que favorece relaciones de comunicación efectiva y oportuna, podría permitirles a los gestores explicarse y comprender cómo mejorar sus actividades, cuáles son los vacíos, ausencias y aciertos de las Políticas Culturales. Esto puede empoderar a las comunidades en las cuales los gestores se mueven o generan.

En el OPC estamos convencidos que todo lo anterior revela el ejercicio de los derechos culturales, los cuales refrendan el bien común por el que el Estado está obligado a velar en una sociedad democrática, y el principio fundamental de toda Política Pública. Los gestores culturales juegan un papel fundamental para el ejercicio del derecho humano denominado acceso y participación en la vida cultura, por ello la recuperación y generación de información y comunicación al respecto permitirá conocer la implementación de las Políticas Culturales tanto para gestores como tomadores de decisiones.

Algunos de los problemas más relevantes que comienzan a vislumbrarse son reforzar el trabajo en las redes sociales para mantener la actualidad de los proyectos y programas de la red y destacar la importancia de la retroalimentación colectiva de los gestores y la participación de la comunidad de base.

No hay una retroalimentación de entidades estatales ni todos los agentes involucrados en el desarrollo de Política Cultural y sus métodos de medición y comunicación. Eso genera dispersión del trabajo de gestores que fragmenta el proyecto estratégico a mediano y largo plazo. Esperamos también que nuestra contribución expuesta en este artículo, incida y lo convierta en un asunto de interés público.

\section{Referencias}

Amozurrutia, J. A., \& Maass Moreno, M. (2013). "Sistemas sociales e investigación interdisciplinaria: una propuesta desde la Cibercultur@". INTERdisciplina, 1(1), 141170. Recuperado a partir de http://revistas.unam.mx/ index.php/inter/article/viewFile/46519/41773

Coelho, T. (2009). Diccionario crítico de Política Cultural. Cultura e imaginario. Barcelona: Gedisa.

González, J. A. (2007). “Cibercultur@y diseño de políticas culturales". En J. L. Mariscal (Ed.), Políticas culturales. Una revisión desde la gestión cultural. (pp. 45-72). Guadalajara: UDG Virtual. Recuperado a partir de http://148.202.167.133/drupal/sites/default/files/Politicas\%20Culturales.pdf

Jiménez, L. (2006). Políticas Culturales en transición. Retos y escenarios de la gestión cultural en México. México: Consejo Nacional para la Cultura y las Artes- Fondo Regional para la Cultura y las Artes Zona Sur.

Maass, M., \& Recaman, A. L. (2014). Dimensión social de la cultura. Gestión cultural para el desarrollo sostenible. (primera). México, D.F.: CONACULTA.

Mariscal, J. L. (2006). "Formación y capacitación de los gestores culturales". Apertura, O(4). Recuperado a partir de http://www.udgvirtual.udg.mx/apertura/index.php/ apertura4/article/view/70

Román, L. (2011). "Una revisión teórica sobre la gestión cultural". Revista Digital de Gestión Cultural, 1(1), 5-17. Recuperado a partir de https://onedrive.live.com/view. aspx?cid=AAED7E2CB767FDA5\&resid=AAED7E2CB767FDA5\%21799\&app=WordPdf

Roth, A.-N. (2002). Políticas Públicas. Formulación, implementación y evaluación. Bogotá: Aurora. 